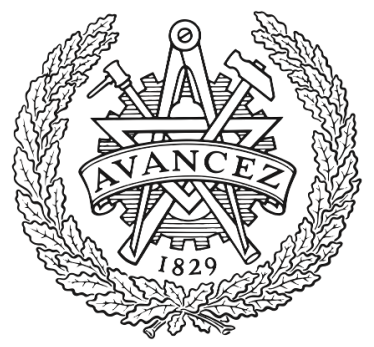

CHALMERS

UNIVERSITY OF TECHNOLOGY

\title{
Analysis and estimation of the maximum circulating current during the parallel operation of reconfigurable battery systems
}

Downloaded from: https://research.chalmers.se, 2023-04-26 14:47 UTC

Citation for the original published paper (version of record):

Han, W., Kersten, A. (2020). Analysis and estimation of the maximum circulating current during the parallel operation of reconfigurable battery systems. 2020 IEEE Transportation Electrification Conference and Expo, ITEC 2020: 229-234. http://dx.doi.org/10.1109/ITEC48692.2020.9161478

N.B. When citing this work, cite the original published paper. 


\title{
Analysis and Estimation of the Maximum Circulating Current during the Parallel Operation of Reconfigurable Battery Systems
}

\author{
Weiji Han, Anton Kersten \\ Department of Electrical Engineering \\ Chalmers University of Technology \\ Gothenburg, Sweden, 41296 \\ Email: weiji.han@chalmers.se, kersten@chalmers.se
}

\begin{abstract}
Reconfigurable battery systems (RBSs) are emerging as a promising solution to safe, efficient, and robust energy storage and delivery through dynamically adjusting the battery connection topology. When the system connection is switched from series to parallel, circulating currents between parallel battery cells/modules can be triggered due to their voltage imbalance. During the hardware design of an RBS, the current rating of associated components, such as batteries, switches, and wires, depends on the maximum circulating currents. Moreover, given a developed RBS, the maximum circulating current also determines whether it is feasible to perform the relevant system reconfiguration. Thus, this paper is focused on modeling and analyzing the current distribution during the series-to-parallel battery reconfiguration and estimating the maximum circulating currents as well as their upper bound under various system states and operating scenarios. A prototype is set up to experimentally verify the effectiveness of the proposed method for estimating the maximum circulating currents.
\end{abstract}

\section{INTRODUCTION}

Battery systems have become an important solution to the large-scale energy storage/delivery in a variety of industrial applications, such as electric vehicles and battery storage power plants serving in power grids. Nowadays, individual battery cells still operate with limited terminal voltage and charging/discharging current. For instance, the emerging Lithium Nickel Manganese Cobalt Oxide battery cells have a typical operating range of $3.0 \mathrm{~V}$ to $4.2 \mathrm{~V}$. To meet the high voltage/current/power requirements in these applications, battery cells are usually connected in series and/or parallel to form battery modules and further packs.

Series-connected battery cells can provide scaled voltage but commonly experience charge imbalance, which could typically lead to reduced charge delivery/storage, accelerated battery aging, and even safety hazards. The parallel battery connection in a pack can help deliver or accept high current, and, meanwhile, it naturally contributes to the voltage balance among parallel battery cells/modules, especially when the battery pack is not externally charged or discharged. Thanks to the emerging technique of battery system reconfiguration, the originally series-connected batteries are possible to be reconnected in parallel. Then, the charge imbalance issue can be mitigated by taking advantage of the self-balancing effect during parallel operation. In comparison to the majority of battery charge balancing methods relying on various external circuit elements [1], [2], the parallel self-balancing, enabled by reconfigurable battery systems (RBSs), can be accomplished only through switches, and thus features lower cost and control complexity.

Unlike conventional battery systems of fixed-configuration, RBSs, normally achieved by connecting two to six switches with each battery cell or module [3], enable the flexible reconfiguration of battery cells or modules. As a result, in addition to the aforementioned battery charge or voltage balance [4], [5], other battery system performance metrics can also be improved [6], such as fault tolerance [7], extended energy delivery [8], programmable terminal voltage [9], and the combined application of batteries of different age or chemistry [10].

When the battery system configuration is switched to parallel connection, as will be illustrated in the circuit experiment results in Section IV, the switch current between parallel batteries demonstrates a rapid increase until a current peak is observed during the initial transient period. After passing the peak, the switch current generally decreases at a much slower pace and finally approaches zero. Then, the first concern for safe parallel operation is to quantify the maximum initial circulating current, which affects not only the current rating of switches in the RBS hardware design but also the feasibility checking of related system reconfiguration during the RBS operation.

Thus, in this work, extensive analysis of the initial circulating currents through switches will be conducted and a model-based analytical method will be proposed to estimate the maximum circulating current and its upper bound under various operating scenarios. An RBS prototype based on $\mathrm{H}$-bridges is set up to facilitate the current analysis as well as to verify the estimation results.

\section{RECONFIGURATION CIRCUIT DESIGN AND SYSTEM MODELING}

\section{A. Circuit design for flexible series and parallel connections}

To illustrate the design of the mutual conversion between series and parallel connections, a small system of only two 
series-connected cells is introduced at first. Clearly, the series and parallel connections of these two cells should be exclusive to avoid any short circuit. Thus, when converting the operation mode from series to parallel, one switch is needed to deactivate the original series connection. Then, to switch the system back to the series operation, two switches need to be inserted to terminate the parallel connection. Therefore, at least three switches are necessary to enable the flexible conversion between the series connection as shown in Fig. 1a and the parallel connection as shown in Fig. 1b. Finally, the two-cell system can be scaled up to incorporate more cells as illustrated in Fig. 1c. In addition to switching the system connection between series and parallel, this reconfigurable system also makes it possible to individually disconnect any fully charged/discharged or faulty cell on the fly, which is very helpful to maintain the continuous and robust system operation. This reconfigurable structure was also applied to supercapacitors to improve the cycle efficiency and capacity utilization in [11].

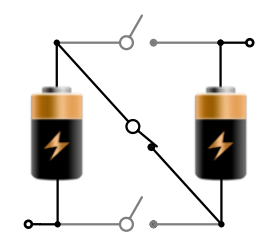

(a)

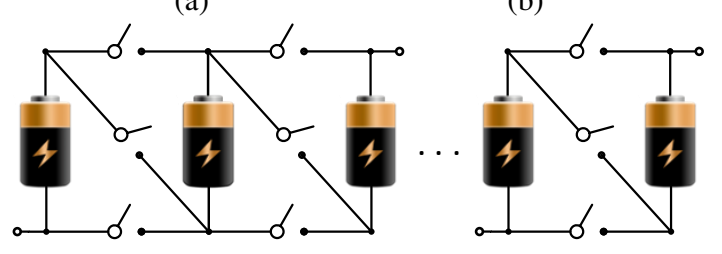

(c)

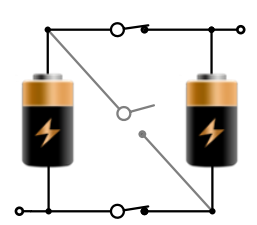

(b)
Fig. 1: Arrangement and working principle of the RBS enabling flexible series and parallel connections of battery cells. (a) Series connection. (b) Parallel connection. (c) RBS with multiple cells.

\section{B. System modeling for parallel operation}

To study the circulating currents during the parallel selfbalancing of this RBS, a generic equivalent circuit diagram is established in Fig. 2.

In this diagram, a typical battery cell model is composed of a voltage source, indicating its open circuit voltage (OCV) denoted by $V_{O C}^{m}, m=1, \ldots, M$, an internal resistance $R_{0}^{m}$ and $N \geq 1$ RC pairs to characterize the dynamic behavior, e.g., diffusion effect. The $n$-th RC pair consists of a resistor $R_{n}^{m}$ and a capacitor $C_{n}^{m}$ with the voltage $V_{n}^{m}, n=1, \ldots, N$. In addition, the MOSFET switch connecting the positive (negative) terminals of the $m$-th and $(m+1)$-th cells is modeled by its onstate resistance denoted by $R_{s+}^{m}\left(R_{s-}^{m}\right), m<M$. Moreover, the resistance of the wires connecting cells and switches, while not explicitly shown in Fig. 2, can be combined into the associated battery internal resistance $R_{0}^{m}$ or the switch resistance, $R_{s+}^{m}$ or $R_{s-}^{m}$.

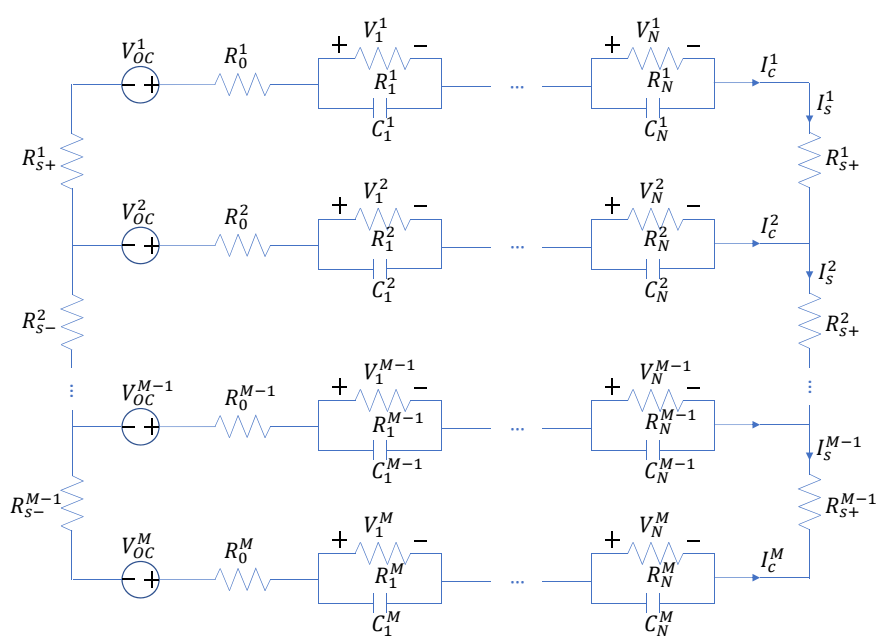

Fig. 2: Equivalent circuit diagram of parallel-connected battery cells by switches.

In this parallel-connected system, the current through the $m$-th cell is denoted by $I_{c}^{m}$, and the circulating currents from the top $m$ cells to the remaining ones are denoted by $I_{s}^{m}$. Based on this equivalent circuit diagram, state-space equations can be established to describe the parallel operation of battery cells. While the formulation of these equations will not be detailed here due to the space limit, the model-based simulation results of four parallel-connected cells through switches without external charging or discharging are shown in Fig. 3. Clearly, the cell OCVs can gradually converge over time, demonstrating the self-balancing effect of parallel connection. Accordingly, the RC pair voltage, cell current, and switch current all approach zero eventually.
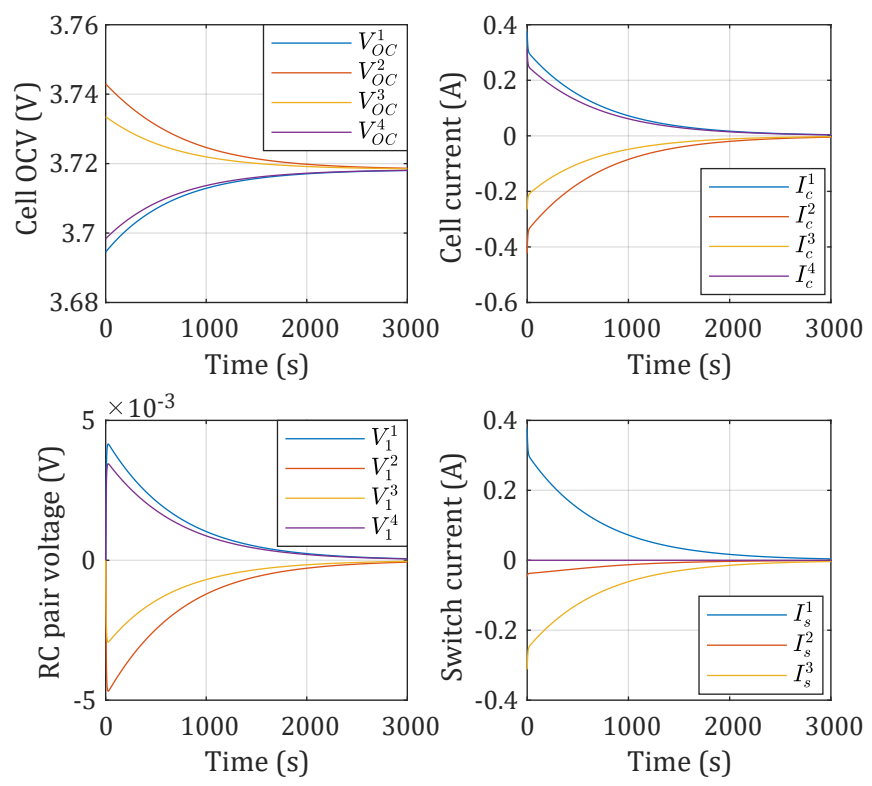

Fig. 3: Simulation results of 4 parallel-connected battery cells. 


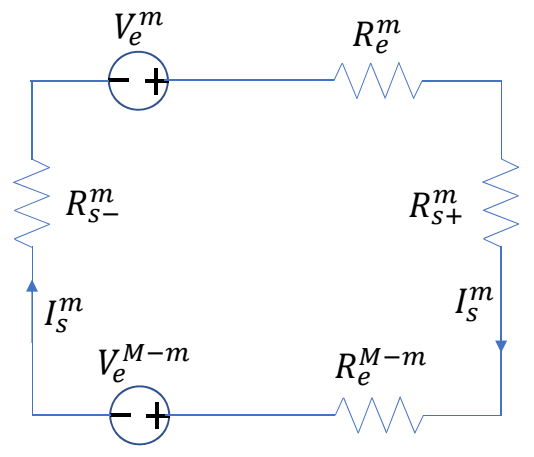

Fig. 4: Modeling the parallel battery system as two two-terminal networks connected in parallel.

\section{Circulating CURRENT ANALysis AND ESTimation}

When the battery cells are reconfigured to parallel connection for, e.g., self-balancing, circulating currents among parallel cells are triggered. Based on the equivalent circuit model in Fig. 2, the circulating currents can reach their peaks almost immediately and then decrease over time as shown in Fig. 3. Then, the maximum circulating current through switches can be expressed as

$$
I_{s}^{\max }=\max _{1 \leq m<M}\left|I_{s}^{m}(t=0)\right| .
$$

Thus, the initial circulating current is extensively studied in this section. For brevity, all the following variables and equations in this section are given at $\mathrm{t}=0$ if not particularly specified.

The self-balancing of parallel-connected battery cells should be implemented when the battery is at rest, e.g., when an electric car is parked in the garage. After sufficient rest, the diffusion process is finished. Then, it can be assumed that each $\mathrm{RC}$ pair's initial voltage approaches zero, i.e.,

$$
V_{n}^{m}=0, \quad m=1, \ldots, M, n=1, \ldots, N .
$$

To simplify the analysis, all cells (switches) are assumed to share the identical battery internal resistance (switch resistance), i.e.,

$$
R_{0}^{m}=R_{c}, R_{s+}^{m}=R_{s-}^{m}=R_{s}, m=1, \ldots, M .
$$

Then, the ratio of the switch resistance and the battery cell resistance is denoted as

$$
\rho=R_{s} / R_{c}
$$

To analyze the circulating current $I_{s}^{m}$ between two adjacent cells, the system can be viewed as two two-terminal networks illustrated in Fig. 4, in which the upper network includes the first to the $m$-th cells and the lower network consists of the remaining cells. The two networks are connected in parallel through the $m$-th pair of switches, and within each network battery cells are still connected in parallel.
Then, for a two-terminal network composed of $K$ parallel cells, the equivalent circuit parameters can be derived as follows.

$R_{e}^{K}=\alpha_{K} R_{c}$,

$V_{e}^{K}= \begin{cases}V_{O C}^{1}, & K=1, \\ \sum_{k=1}^{K-1}\left(\prod_{j=k+1}^{K}\left(1-\alpha_{j}\right) \alpha_{k} V_{O C}^{k}\right)+\alpha_{K} V_{O C}^{K}, & K>1,\end{cases}$

$\alpha_{k}= \begin{cases}1, & \text { if } k=1 \\ 1-\frac{1}{\alpha_{k-1}+2 \rho+1}, & \text { if } k>1 .\end{cases}$

Based on the equivalent circuit in Fig. 4 with parameters given by (5), (6), and (7), the magnitude of the initial circulating current between two networks can be calculated by

$$
\left|I_{s}^{m}\right|=\frac{\left|V_{e}^{m}-V_{e}^{M-m}\right|}{R_{e}^{m}+R_{e}^{M-m}+2 \rho R_{c}}=\frac{\left|V_{e}^{m}-V_{e}^{M-m}\right|}{\left(\alpha_{m}+\alpha_{M-m}+2 \rho\right) R_{c}} .
$$

Then, the maximum circulating current $I_{s}^{\max }$ can be identified using (1).

For the current rating of switches, it is necessary to evaluate the upper bound of circulating currents under all possible operating scenarios, denoted by $I_{s}^{u b}$. According to (1) and (8), this upper bound can be estimated by

$$
\begin{aligned}
I_{s}^{\text {max }} \leq I_{s}^{u b} & =\frac{V_{O C}^{\text {ulim }}-V_{O C}^{\text {llim }}}{\left(\min _{1 \leq m<M}\left\{\alpha_{m}+\alpha_{M-m}\right\}+2 \rho\right) R_{c}}, \\
& =\frac{V_{O C}^{r l i m}}{\min _{1 \leq m<M}\left\{\alpha_{m}+\alpha_{M-m}\right\} R_{c}+2 R_{s}} .
\end{aligned}
$$

where $V_{O C}^{\text {ulim }}$ and $V_{O C}^{\text {llim }}$ are the specified upper and lower limits of cell OCVs, respectively, and, thus, the corresponding battery cell OCV range limit is $V_{O C}^{\text {rlim }}=V_{O C}^{u l i m}-V_{O C}^{\text {llim }}$.

The estimated upper bound of switch current plays an important role in selecting appropriate battery cells and switches when setting up an RBS. Using this expression in (9), the upper bound of RBS switch current, i.e., the maximum circulation current, will be evaluated based on parameter values collected from the following real battery cells and switches.

The 18650 Lithium-ion battery cells feature an internal resistance of tens of milliohms depending on the cell SOC, temperature, and age, etc. For instance, the four battery cells tested in [12] have an internal resistance ranging from $33 \mathrm{~m} \Omega$ to $47 \mathrm{~m} \Omega$. Besides, the MOSFET switch's on-resistance $R_{s}$ varies from several to tens of milliohms in various designs and applications, e.g., $R_{s}=8.4 \mathrm{~m} \Omega$ in [13] and $R_{s}=47 \mathrm{~m} \Omega$ in [14]. Then, taking the average battery internal resistance in [12], i.e., $R_{c}=40 \mathrm{~m} \Omega$, and the switch resistances in [13] and [14] for example, and assuming the number of parallel cells $M$ is very large, the switch current upper bounds under various battery OCV range limits are evaluated using (9) and the results are listed in Table I. The switch current upper limits specified by manufacturers are $40 \mathrm{~A}$ in [13] and $30 \mathrm{~A}$ in [14]. It can be seen that even in certain extreme cases with very large 
TABLE I: The upper bound of switch current evaluated at various battery OCV range limits.

\begin{tabular}{l|c|c}
\hline \hline & $\rho=0.21[12],[13]$ & $\rho=1.175[12],[14]$ \\
\hline$V_{O C}^{r l i m}=0.1 \mathrm{~V}$ & $1.835 \mathrm{~A}$ & $0.647 \mathrm{~A}$ \\
\hline$V_{O C}^{r l i m}=0.5 \mathrm{~V}$ & $9.174 \mathrm{~A}$ & $3.236 \mathrm{~A}$ \\
\hline$V_{O C}^{r l i m}=1.2 \mathrm{~V}$ & $22.018 \mathrm{~A}$ & $7.766 \mathrm{~A}$ \\
\hline \hline
\end{tabular}

cell OCV range up to $1.2 \mathrm{~V}$, the switch current upper bounds are still lower than these specified limits of switch currents, indicating that these combinations of battery cells and switches are applicable to the RBS design.

Remark 3.1: The switch current upper bound needs to be evaluated before constructing the reconfigurable battery systems in Fig. 1. To test a large number of operating conditions, circuit experiments are very inefficient in time and energy, and thus model-based simulation or analysis are preferred. As compared to simulating various working scenarios, the proposed analytical expression in (9) can provide the same estimation results for the maximum circulating current but requires much less computational efforts. The effectiveness of the proposed method will be evaluated through circuit experiments in Section IV.

Remark 3.2: It is practically simple to evaluate the switch current upper bound by (9) since only two parameter values are needed, i.e., the battery internal resistance and the switch on-resistance. For new cells and switches, these parameters can be readily looked up from the datasheets provided by manufacturers. As battery cells age over time, the battery internal resistance $R_{c}$ generally increases, and, thus $\rho$ decreases. According to (9), it can be proved that the cell degradation over time contributes to a decreasing upper bound. Then, the upper bound calculated based on new cells becomes an overestimate for aged cells. This overestimate is a little conservative but can still ensure the safe operation, indicating that these switches do not need to be upgraded due to the battery degradation throughout the entire lifespan.

\section{EXPERIMENTAL VALIDATION}

\section{A. Circuit implementation based on H-bridge modules}

To verify the effectiveness of the proposed estimation method for the maximum circulating current, a prototype of the RBS design in Fig. 1 was set up as shown in Fig. 5a based on classic H-bridge modules, which are widely used in many power electronic converters. Fig. 5b shows the schematic of the reconfigurable battery arrangement using H-bridges. More detailed switch arrangement for series and parallel connections along with their corresponding gate signals are presented in Fig. 6. The switches of each H-bridge are operated in pairs, and the switches of each half-bridge must be complementary operated. Besides, for safety reasons, all battery cells are equipped with fuses.

Based on the battery and MOSFET switch datasheets and experimental measurements, the following parameter values are extracted for estimating the maximum switch current. $R_{c}=71.5 \mathrm{~m} \Omega$ is composed of the battery internal resistance

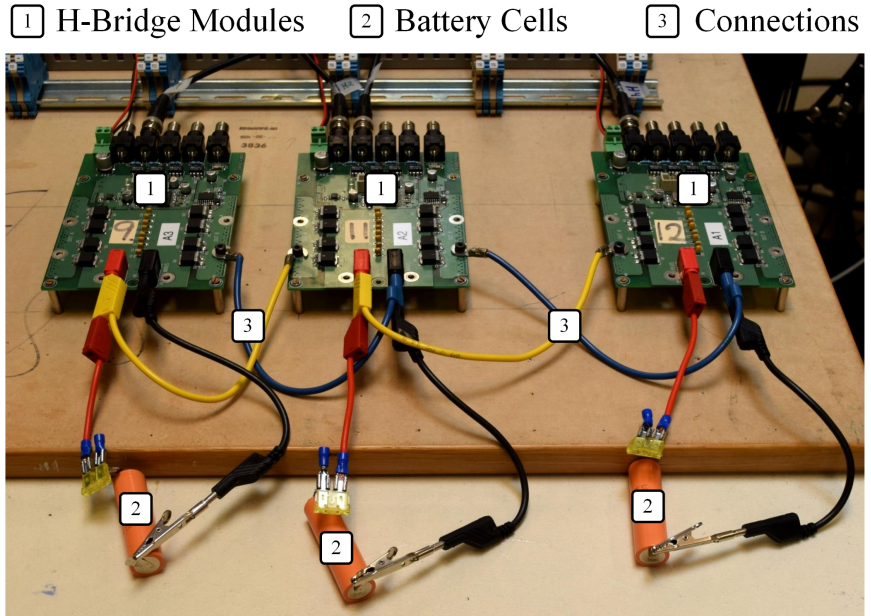

(a)

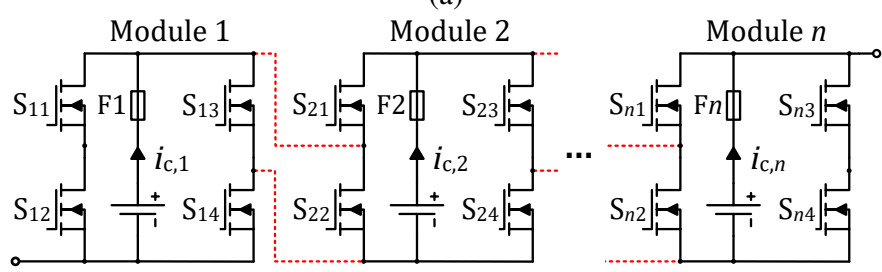

(b)

Fig. 5: (a) Laboratory setup with three H-bridge modules. (b) Schematic of the RBS.

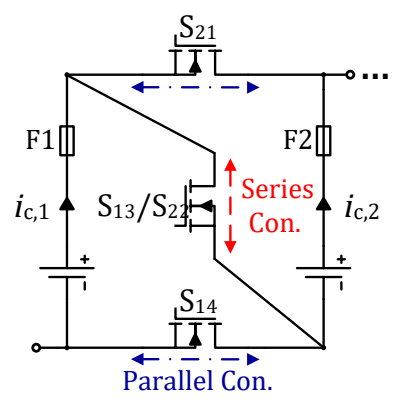

(a)

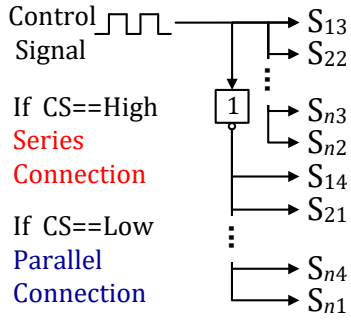

(b)
Fig. 6: (a) Topology arrangement using H-bridge modules. (b) Gate signals for series and parallel connection.

$R_{0}=62.1 \mathrm{~m} \Omega$, the connection wire's resistance $6 \mathrm{~m} \Omega$, and the fuse's resistance $3.4 \mathrm{~m} \Omega$. Besides, $R_{s}=5.3 \mathrm{~m} \Omega$ consists of the switch on-state resistance $1 \mathrm{~m} \Omega$ and all the resistance along the conduction path on the PCB. Then, based on (4), the switch-battery resistance ratio is $\rho \approx 0.0741$. In addition, the battery OCV limits are set to $V_{O C}^{\text {llim }}=3 \mathrm{~V}, V_{O C}^{\text {ulim }}=4.2 \mathrm{~V}$, and $V_{O C}^{r l i m}=1.2 \mathrm{~V}$.

\section{B. Experiments for maximum circulating current}

To evaluate the maximum circulating current as well as its upper bound, a harsh operating scenario of two cells with a large initial OCV difference of $1.1 \mathrm{~V}$ was tested, and the results are shown in Fig. 7. Once the cells get connected in parallel at 
$\mathrm{t}=0$, the circulating current, i.e., switch current, (marked by blue dots) reaches its peak very quickly, within about $110 \mu \mathrm{s}$. When zooming in this current rising period, some oscillations can be observed due to the resonance caused by the parasitic inductance and capacitance on associated circuit elements. The spikes during oscillation are not the focus of this study since they typically appear for less than $1 \mu$ s and almost cause no damage to the battery cells and switches. After the peak, the switch current decreases over time at a much slower pace. As compared in Fig. 7, the estimated $I_{s}^{\max }$ (green dash-dot line) is quite close to the experimental $I_{s}^{\max }$ (blue dash line) and both fall below the estimated upper bound (red dash-dot line). These results verify the accuracy of the estimated maximum circulating current and the effectiveness of the estimated upper bound.

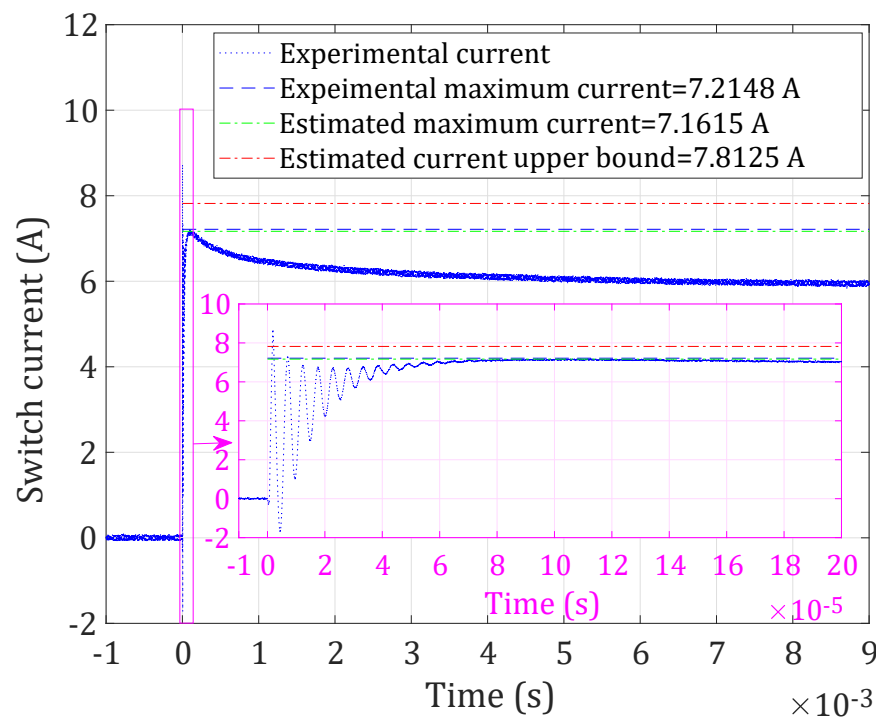

Fig. 7: Comparison of the estimated maximum circulating current and its upper bound with experimental results for a two-cell system with initial cell OCVs $4.17 \mathrm{~V}$ and $3.07 \mathrm{~V}$.

In addition, another test was performed on the built three-cell RBS following the similar procedure but with a narrower initial cell OCV range of $0.379 \mathrm{~V}$. The experimental and estimation results are compared in Fig. 8. The estimated $I_{s}^{\max }$ by (1) and (8) is similar to the experimental result but a little lower. Assume the current upper bound is still based on the OCV range limit $V_{O C}^{\text {rlim }}=1.2 \mathrm{~V}$, much larger than the given battery $\mathrm{OCV}$ range of $1.2 \mathrm{~V}$ in this test. Then, the current upper bound is expected to be much higher than the observed maximum switch current. This is validated in Fig. 8 where the estimated switch current upper bound is more than five times as much as the measured $I_{s}^{\max }$. Therefore, the estimated switch current upper bound is reliable for the switch current rating.

\section{CONCLUSIONS AND FUTURE WORK}

RBSs enable the flexible switch between series and parallel connections and, hence, provide a new solution to advanced

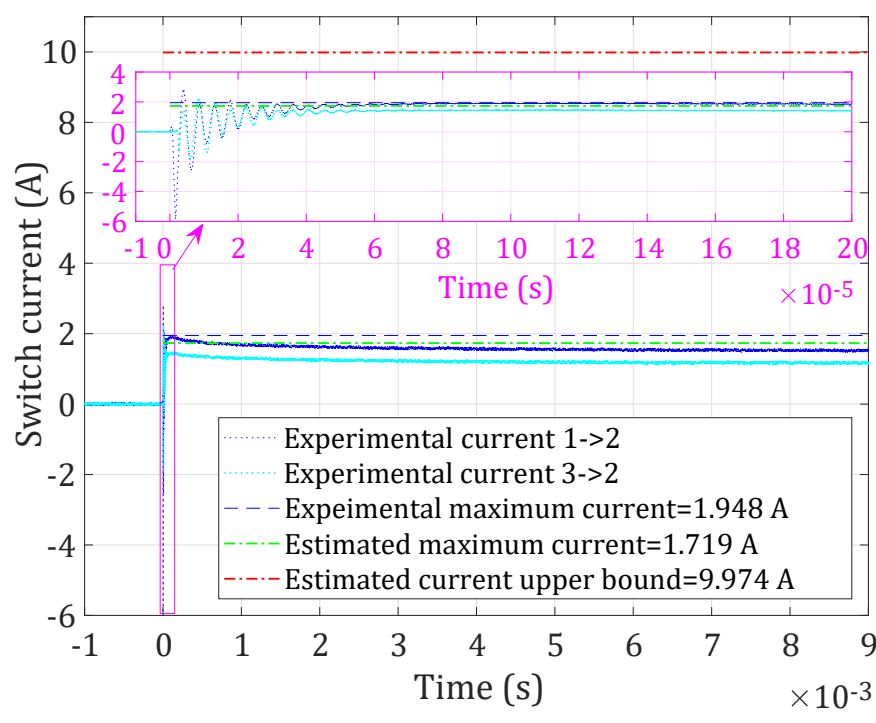

Fig. 8: Comparison of the estimated maximum switch current and switch current upper bound with the experimental currents for a three-cell system with initial cell OCVs $4.083 \mathrm{~V}, 3.704 \mathrm{~V}$, and $4.074 \mathrm{~V}$.

battery management and control. When an RBS is reconfigured to parallel battery operation, circulating currents arise and its peak determines the current rating of switches applied in the RBS and also the feasibility of such parallel operation. Thus, this paper has been focused on analyzing and estimating the circulating current among parallel batteries. Specifically, the maximum circulating current and its upper bound have been analytically estimated and the estimation accuracy has been validated by circuit experiments. In future work, the analysis and estimation will be extended to larger-scale systems, and the external charging or discharging pack current will also be taken into account.

\section{REFERENCES}

[1] W. Han, L. Zhang, and Y. Han, "Mathematical modeling, performance analysis and control of battery equalization systems: Review and recent developments," in Advances in Battery Manufacturing, Services, and Management Systems, J. Li, S. Zhou, and Y. Han, Eds. New York: Wiley-IEEE Press, 2016, ch. 12, pp. 281-301.

[2] W. Han, C. Zou, C. Zhou, and L. Zhang, "Estimation of cell SOC evolution and system performance in module-based battery charge equalization systems," IEEE Trans. Smart Grid, vol. 10, no. 5, pp. 47174728, Sept. 2019.

[3] S. Ci, N. Lin, and D. Wu, "Reconfigurable Battery Techniques and Systems: A Survey," IEEE Access, vol. 4, pp. 1175-1189, 2016.

[4] W. Han and L. Zhang, "Battery cell reconfiguration to expedite charge equalization in series-connected battery systems," IEEE Robot. Autom. Lett., vol. 3, no. 1, pp. 22-28, Jan. 2018.

[5] W. Han, C. Zou, L. Zhang, Q. Ouyang, and T. Wik, "Near-fastest battery balancing by cell/module reconfiguration," IEEE Trans. Smart Grid, vol. 10, no. 6, pp. 6954-6964, Nov. 2019.

[6] W. Han, T. Wik, A. Kersten, G. Dong, and C. Zou, "Next-Generation Battery Management Systems: Dynamic Reconfiguration," IEEE Ind. Electron. Mag., 2020, accepted.

[7] T. Kim, W. Qiao, and L. Qu, "Power electronics-enabled self-X multicell batteries: A design toward smart batteries," IEEE Trans. Power Electron. vol. 27 , no. 11 , pp. 4723-4733, Nov. 2012. 
[8] Z. Zhang, Y. Cai, Y. Zhang, D. Gu, and Y. Liu, "A distributed architecture based on microbank modules with self-reconfiguration control to improve the energy efficiency in the battery energy storage system," IEEE Trans. Power Electron., vol. 31, no. 1, pp. 304-317, Jan. 2016.

[9] M. Alahmad, H. Hess, M. Mojarradi, W. West, and J. Whitacre, "Battery switch array system with application for JPL's rechargeable micro-scale batteries," J. Power Sources, vol. 177, no. 2, pp. 566-578, 2008.

[10] A. Badam, R. Chandra, J. Dutra, A. Ferrese, S. Hodges, P. Hu, J. Meinershagen, T. Moscibroda, B. Priyantha, and E. Skiani, "Software defined batteries," in Proc. ACM 25th Symp. Operating Syst. Principles, 2015, pp. 215-229.

[11] Y. Kim, S. Park, Y. Wang, Q. Xie, N. Chang, M. Poncino, and M. Pedram, "Balanced reconfiguration of storage banks in a hybrid electrical energy storage system," in IEEE/ACM Int. Conf. Comput.Aided Design, Nov. 2011, pp. 624-631.

[12] D. Wang, Y. Bao, and J. Shi, "Online lithium-ion battery internal resistance measurement application in state-of-charge estimation using the extended kalman filter," Energies, vol. 10, no. 9, 2017.

[13] Infineon Technologies AG, "Infineon optimos power mosfet ipz40n04s58r4 datasheet," https://www.infineon.com/dgdl/Infineon-IPZ40N04S5 8R4-DS-v01_00-EN.pdf?fileId=5546d462584d1d4a0158d8a817e4652a, July 2015, (Accessed on 07/09/2019).

[14] Fairchild Semiconductor Corporation, "30A, 60V, ESD Rated, 0.047 Ohm, logic level N-Channel power MOSFETs," https://www.sparkfun.com/datasheets/Components/General/RFP30N06L E.pdf, 2004, (Accessed on 07/09/2019). 